\title{
(C) OPEN ACCESS \\ Less frequently mutated genes in colorectal cancer: evidences from next-generation sequencing of 653 routine cases
}

\author{
Umberto Malapelle, ${ }^{1}$ Pasquale Pisapia, ${ }_{1}^{1}$ Roberta Sgariglia, ${ }^{1}$ Elena Vigliar, ${ }_{1}^{1}$ \\ Maria Biglietto, ${ }^{2}$ Chiara Carlomagno, ${ }^{3}$ Giuseppe Giuffrè, ${ }^{4}$ Claudio Bellevicine, ${ }^{1}$ \\ Giancarlo Troncone ${ }^{1}$
}

- Additional material is published online only. To view please visit the journal online (http://dx.doi.org/10.1136/ jclinpath-2015-203403)

${ }^{1}$ Department of Public Health, University of Naples Federico II, Naples, Italy

${ }^{2}$ Department of Oncology، AORN Cardarelli, Naples, Italy ${ }^{3}$ Department of Surgical and Clinical Medicine, University of Naples Federico II, Naples, Italy

${ }^{4}$ Department of "Patologia Umana dell'Adulto e dell'età evolutiva, G.Barresi", University of Messina, Messina, Italy

\section{Correspondence to}

Professor Giancarlo Troncone, Department of Public Health University of Naples Federico II, via Sergio Pansini 5 ,

Naples 1-80131, Italy; giancarlo.troncone@unina.it

UM and PP contributed equally.

Received 15 September 2015 Revised 28 December 2015 Accepted 29 December 2015 Published Online First 21 January 2016

\section{CrossMark}

\section{To cite: Malapelle $U$,}

Pisapia P, Sgariglia $R$, et al.

J Clin Pathol 2016;69:767-

771.

\section{ABSTRACT}

Aims The incidence of RAS/RAF/PISKA and TP53 gene mutations in colorectal cancer (CRC) is well established. Less information, however, is available on other components of the CRC genomic landscape, which are potential CRC prognostic/predictive markers.

Methods Following a previous validation study, ionsemiconductor next-generation sequencing (NGS) was employed to process 653 routine CRC samples by a multiplex PCR targeting 91 hotspot regions in 22 CRC significant genes.

Results A total of 796 somatic mutations in 499 (76.4\%) tumours were detected. Besides RAS/RAF/PI3KA and TP53, other 12 genes showed at least one mutation including FBXW7 (6\%), PTEN (2.8\%), SMAD4 (2.1\%), EGFR (1.2\%), CTNNB1 (1.1\%), AKT1 (0.9\%), STK11 $(0.8 \%), \operatorname{ERBB2}(0.6 \%), \operatorname{ERBB} 4(0.6 \%), \operatorname{ALK}(0.2 \%)$, MAP2K1 (0.2\%) and NOTCH1 (0.2\%).

Conclusions In a routine diagnostic setting, NGS had the potential to generate robust and comprehensive genetic information also including less frequently mutated genes potentially relevant for prognostic assessments or for actionable treatments.

\section{INTRODUCTION}

Antiepidermal growth factor receptor (EGFR) therapy is not effective in patients with metastatic colorectal cancer (CRC) harbouring mutations at codons 12 and 13 in KRAS exon $2 .^{1}$ More recent evidences showed that the so-called expanded RAS mutations (exon 3 and exon 4 of KRAS and exons 2, 3 and 4 of NRAS) also have negative predictive value. ${ }^{2}$ The extension of community KRAS testing to all $R A S$ mutations favoured the implementation of multitarget testing methodologies. Nextgeneration sequencing (NGS), matched with multiplex capture of targeted gene regions and analysed by bioinformatics tools, enables the simultaneous detection of multiple mutations in multiple genes. The development of affordable benchtop sequencers, such as the Ion Torrent Personal Genome Machine (PGM; Life Technologies, Carlsbad), and of relatively small, focused gene panels, such as the Ion AmpliSeq Colon and Lung Cancer Panel, ${ }^{3}$ enabled our laboratory to adopt NGS as a stand-alone diagnostic test to genotype KRAS NRAS and $B R A F^{4}$ In a previous validation study, all point mutations detected in these genes by Sanger sequencing were also correctly identified by NGS. ${ }^{4}$ The latter, however, proved to be more sensitive, and, remarkably, less costly. ${ }^{4}$
NGS may also identify rarer patient-specific somatic mutations. The latter are of unclear significance, as their incidence rates have not been established with certainty. In fact, while there is a wealth of data regarding RAS/RAF/PI3KA and TP53 gene mutations, the information on less frequently mutated genes is mostly derived by the genomic scale analysis of a limited number of CRC samples. ${ }^{5}$ Conversely, in its daily diagnostic practice, our laboratory, an Italian accredited reference centre for RAS testing, has generated a large database of CRC samples sequenced with the PGM/ Colon Lung Cancer Panel, whose interrogation can be useful to better define the incidence rate of rare mutations. Thus, besides KRAS, NRAS, BRAF PIK3CA and TP53 alterations, this paper focuses on mutations occurring in other receptor tyrosine kinase (RTK) genes (ALK, EGFR, ERBB2, ERBB4, FGFR1, FGFR2, FGFR3, MET, DDR2), in RTK signalling genes (AKT1, PTEN, MAP2K1, STK11) and in other well-known cancer-related genes (NOTCH1, CTNNB1, SMAD4, FBXW7).

\section{METHODS}

\section{Patients and samples}

This study includes a series of 653 CRC tissue samples (398 men and 255 women) referred from 18 institutions located all over South Italy between January 2014 and March 2015. Mean patient age was 66.8 years (range, 29-96 years). Following current international guidelines, one single tumour sample was tested for each patient. ${ }^{6}$

\section{NGS analysis}

Tumour cell enrichment, DNA extraction and NGS analysis on the Ion Torrent PGM by using the AmpliSeq Colon and Lung Cancer panel were performed, as previously described, ${ }^{4}$ and detailed in online supplementary information (file 1). The Torrent Suite V.4.0 analysis pipeline was used to assess the sequencing data and to perform adapter trimming, alignment QC and base calling. Single-nucleotide polymorphisms, insertions and deletions (del) were identified using a Torrent Variant Caller plug-in (V.4.0-r76860), optimised for low-frequency variants assessment. The criteria for evaluation of any variant as reportable were the following: minimum coverage depth of $100 \times$, minimum variant frequency of $5 \%$ and confirmation by the Integrative Genomics Viewer visual inspection. Sequence variants, deemed real and reportable 
Table 1 Twenty-two multiple gene mutation analysis by the lon Torrent AmpliSeq Colon and Lung Cancer Panel in routine samples of colorectal cancer

\begin{tabular}{ll}
\hline Total cases analysed & $\mathrm{n}=653$ \\
Wild type in all 22 gene analysed & $\mathrm{n}=154(23.6 \%)$ \\
Mutated at $\geq 1$ of 22 genes analysed & $\mathrm{n}=499(76.4 \%)$ \\
Total mutations & $\mathrm{n}=796$ \\
Mutated genes & $17 / 22$ \\
\hline
\end{tabular}

by criteria listed above, were further assessed by the ClinVar Database (http://www.ncbi.nlm.nih.gov/clinvar/, last accessed 30 November 2015) for classifying a genetic alteration as germline or somatic.

\section{RESULTS}

One or more gene mutations were detected in 499/653 (76.4\%) tumours in 17 of the 22 genes included in the panel (table 1), for a total of 796 mutations that are listed in online supplementary information (file 2). A representative case is reported in figure 1. Only three genes (DDR2, FGFR1 and FGFR2) did not harbour any alteration, while two genes (FGFR3 and MET) only harboured germline variants as reported in online supplementary information (file 3). Single mutations were found in 274 patients (41.9\%), double mutations in 177 patients $(27.1 \%)$ and 3 or more mutations were found in 48 patients $(7.4 \%)$. Coexisting mutations in different genes are reported in online supplementary table $\mathrm{S} 1$.

Mutations occurred in TP53 $(\mathrm{n}=240 ; 38.8 \%)$, KRAS $(\mathrm{n}=247 ; 37.8 \%)$, NRAS $(\mathrm{n}=30 ; 4.6 \%)$ and BRAF $(\mathrm{n}=63$; 9.6\%). KRAS and NRAS mutations were mutually exclusive. KRAS and NRAS coexisted with BRAF mutations in four and in one instances, respectively. In most of these cases (4/5), BRAF mutations occurred outside of codon 600. PIK3CA gene mutations occurred in $98(15 \%)$ cases. More frequently, PIK3CA mutations were detected together with other gene mutations; PIK3CA was the only mutated gene in $15 / 98$ (15.3\%) samples.

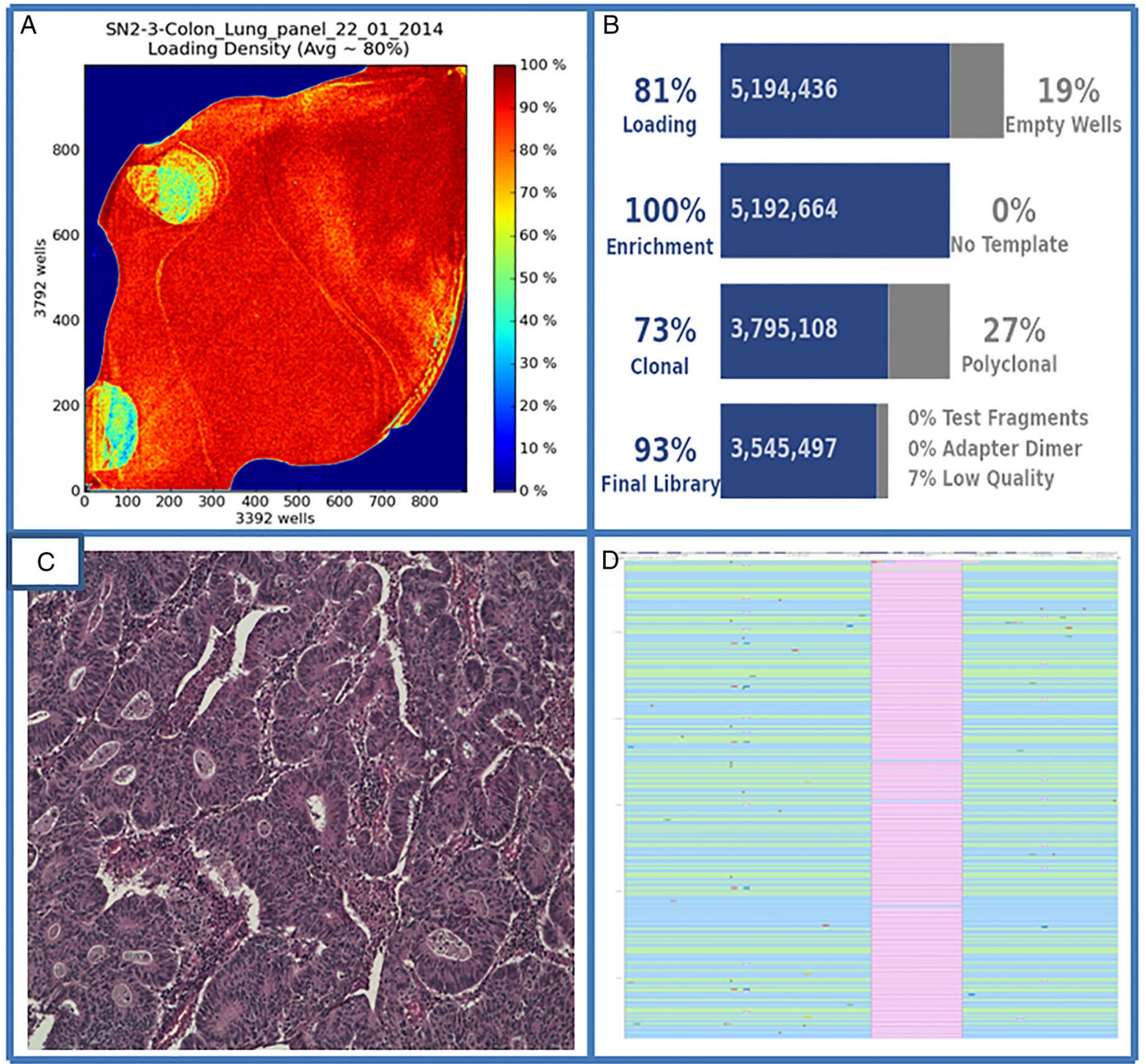

Figure 1 Loading density (A) and performance parameters (B) of an lon Torrent sequencing run, carried out using a 316 chip, are shown. DNA extracted from the colorectal cancer (CRC) shown in (C) harboured an epidermal growth factor receptor p.E746_A750delELREA mutation. (D) was observed with a Genome Brower web app. 
Table 2 Number and percentage of cases of each gene sequenced by the Ion Torrent AmpliSeq Colon and Lung Cancer Panel

\begin{tabular}{lc} 
Gene & Number of mutated cases (\%) \\
\hline KRAS & $247^{*}(37.8 \%)$ \\
TP53 & $240 \dagger(36.8 \%)$ \\
PIK3CA & $98 \ddagger(15 \%)$ \\
BRAF & $63(9.6 \%)$ \\
FBXW7 & $39(6 \%)$ \\
NRAS & $30(4.6 \%)$ \\
PTEN & $18(2.8 \%)$ \\
SMAD4 & $14(2.1 \%)$ \\
EGFR & $8(1.2 \%)$ \\
CTNNB1 & $7(1.1 \%)$ \\
AKT1 & $6(0.9 \%)$ \\
STK11 & $5(0.8 \%)$ \\
ERBB4 & $4(0.6 \%)$ \\
ERBB2 & $4(0.6 \%)$ \\
NOTCH1 & $1(0.2 \%)$ \\
ALK & $1(0.2 \%)$ \\
MAP2K1 & $1(0.2 \%)$ \\
\hline Note: DDR2, FGFR1, FGFR2, FGFR3 and MET genes did not harbour any alteration. \\
${ }^{4} 4 / 247$ cases harboured 2 KRAS mutations. \\
†5/240 cases harboured 2 TP53 mutations. \\
\$1/98 cases harboured 2 PIK3CA mutations.
\end{tabular}

Number and percentage of mutated cases of each gene are reported in table 2 and exons and codons involved are detailed in online supplementary information (file 4).

Besides RAS/RAF/PI3KA and TP53 gene mutations, the Ion AmpliSeq Colon and Lung Cancer Panel provided information on additional targets, such as RTK genes, RTK signalling genes and other well-known cancer-related genes, as it follows.

\section{RTK gene mutations}

ALK: in one case $(0.2 \%)$ the p.L1196M mutation was detected in association with two mutations of the TP53 gene. EGFR: mutations occurred in eight (1.2\%) cases, with exon 19 deletion evident in four instances $(n=3$ p.E746_E749delELRE; $n=1$ p.E746_A750delELREA, as shown in figure 1). Most cases (7/8) were associated with other gene alterations; in particular, five cases harboured a KRAS mutation. ERBB2: mutations occurred in four $(0.6 \%)$ cases, with the V842I being detected in three instances. ERBB4: mutations occurred in four cases $(0.6 \%)$.

\section{RTK signalling genes mutations}

AKT1: the E17K mutation occurred in six cases (0.9\%). PTEN: mutations occurred in $18(2.8 \%)$ cases. MAP2K1: in one case $(0.2 \%)$ the $\mathrm{K} 57 \mathrm{~N}$ mutation was associated with PIK3CA mutation. STK11: mutations occurred in five cases $(0.8 \%)$.

\section{Other cancer-related genes}

NOTCH1: mutation occurred in one case $(0.2 \%)$ and remarkably this case had five additional gene mutations occurring in TP53, KRAS, PTEN, ERBB4 and PIK3CA. CTNNB1: mutations were detected in seven cases (1.1\%), being always associated with at least one other concurrent mutation. In particular, CTNNB1 mutations were consistently associated with the constitutive activation of the $R A F / M E K / E R K$ pathway by either $K R A S$ $(\mathrm{n}=4)$ or $B R A F(\mathrm{n}=3)$ concurrent mutations. SMAD4: mutations were found in 14/653 (2.1\%) samples, and in combination with other mutations (9/14). FBXW7: mutations were identified in $39 / 653$ patients $(6 \%)$, singly $(n=7)$ and associated with KRAS $(n=20)$.

\section{DISCUSSION}

This study evaluated in CRC routine samples a broad set of genes for mutational events. Previous evidences regarding the $R A S / R A F / P I 3 K A$ gene were confirmed. KRAS and NRAS mutations were always mutually exclusive, ${ }^{5}$ whereas occasionally $B R A F$ (mostly no V600E) mutations coexisted with an RAS gene alteration. ${ }^{7}$ The frequent association of PIK3CA mutations with the $R A S / R A F$ alterations was also confirmed. ${ }^{5}$ Our data straighten the view that the simple distinction of tumours in RAS, BRAF or PIK3CA does not apply to CRC with combined $R A S / R A F$ genetic changes. ${ }^{7}$ We also confirmed that one of the most frequently mutated genes in CRC is TP53, whose mutation rate in our study was $38.8 \%$.

Additional information was generated on other potentially actionable components of the CRC genomic landscape, such as RTK genes. Remarkably, the ALK p.L1196M gatekeeper mutation, which confers high-level resistance to crizotinib in lung cancer, was for the first time detected in CRC. EGFR mutations were also detected, as shown in figure 1 , and their mutation rate $(1.2 \%)$ was lower than that $(4.5 \%)$ reported in the Tumor Cancer Genome Atlas (TCGA). ${ }^{5}$ While KRAS and EGFR mutations are normally exclusive, concomitant KRAS and EGFR mutations were also detected (see online supplementary table S1), confirming previous NGS findings. ${ }^{8}$ Other mutations include those involving ERBB2; in particular, the V842I ERBB2 mutation associated with breast cancer ${ }^{9}$ was detected in three instances. Remarkably, in CRC preclinical models HER2 mutations were resistant to cetuximab and panitumumab and responsive to second-generation HER2/EGFR irreversible tyrosine, afatinib and neratinib. ${ }^{10}$ Clinical trials targeting HER2 activating mutations in metastatic CRC are ongoing. ${ }^{11}$ ERBB4 mutations occurring in $0.6 \%$ of the cases have an uncertain prognostic significance. In fact, the TCGA data set indicated a survival disadvantage in colorectal carcinoma with ERBB4, ${ }^{5} 12$ whereas another study showed that the ERBB4 mutant clones are not selected in metastatic spread. ${ }^{13}$

A number of rare mutations occurring in the PI3K/AKT/ $m T O R$ pathway are potentially actionable. As an example, AKT1 mutations were associated with primary resistance to anti-EGFR therapy. ${ }^{14}$ In our study, AKT1 was mutated in $0.9 \%$ of cases, being mutually exclusive with PIK3CA alterations, as previously shown. ${ }^{14}$ The recent association between E17K AKT1 and tumours with mucinous morphology was observed only in one of our six cases. ${ }^{14}$ Previous studies showed a wide range of PTEN mutation rates $\left(0.7 \%{ }^{15}\right.$ to $\left.6 \%{ }^{16}\right)$. In our study, the mutation rate of PTEN was $2.8 \%$. Interestingly, a total of 11 different mutations were found, according to the notion that mutations in tumour suppressor genes do not strongly cluster in single mutational hot spot. ${ }^{17}$ Another RTK signalling gene included in our panel is the STK11 gene. We confirm that somatic STK11 mutations rarely occur in somatic CRC (0.8\%). ${ }^{18}$ Earlier studies reported that STK11 mutant neoplasms had alterations in nucleotide metabolism that confer hypersensitivity to deoxythymidylate kinase inhibition, proposing that deoxythymidylate kinase is a possible therapeutic target. ${ }^{19}$

Interestingly, CTNNB1 mutations detected in $1.1 \%$ of the cases were always associated with at least one other concurrent mutation (see online supplementary table S1). In particular, CTNNB1 mutations were consistently associated with the constitutive activation of the $R A F / M E K / E R K$ pathway by 
either $\operatorname{KRAS}(\mathrm{n}=4)$ or BRAF $(\mathrm{n}=3)$ concurrent mutations, in keeping with the notion that CTNNB1 mutations are early events in CRC carcinogenesis. ${ }^{20}$ Conversely, our data confirm that the occurrence of SMAD4 mutations $(2.1 \%)$ is a late event. $^{21}$ In fact, in our study $64.3 \%$ of SMAD4 mutations occurred in combination with other alterations. SMAD4 loss of function was associated with a worse prognosis and decreased disease-free survival and with resistance to 5fluorouracil chemotherapy. ${ }^{22}$ In this present study, FBXW7, a major tumour suppressor gene crucial in promoting exit from the cell cycle, was mutated in $6 \%$ of cases, which is in line with the estimated 9\% of CRCs containing FBXW7 mutations. $^{24} 25$ Preclinical data have suggested that inactivating mutations of FBXW7 could predict sensitivity either to the $m T O R$ inhibitor rapamycin, ${ }^{26}$ or to the histone deacetylase inhibitor MS-275. ${ }^{27}$ Noteworthy, as it was shown in previous reports FBXW7 were often (51.2\%) associated with KRAS mutations. $^{28} 29$ Interestingly, concurrent molecular aberrations can contribute to limited therapeutic efficacy of $m T O R$ inhibitors in the presence of FBXW7 mutations.

Certain genes included in our panel, such as $M A P 2 K 1$, may have a future role in sensitivity, resistance or both, to a variety of preclinical drugs. Targeting of NOTCH signalling may be of therapeutic value in colon cancers, as activating mutations in NOTCH-1 have been previously reported in colon cancer. ${ }^{30}$ In our study NOTCH mutation occurred in one case $(0.2 \%)$ and remarkably this case had five additional gene mutations occurring in TP53, KRAS, PTEN, ERBB4 and PIK3CA.

In conclusion, our data confirm that CRCs consist of a group of heterogeneous disorders with a large number of diverse sets of genetic changes in oncogenes and tumour suppressor genes. In a routine diagnostic setting, the Ion PGM and AmpliSeq colon and Lung Cancer Panel had the potential to exploit even a low-input DNA to uncover multiple common mutations simultaneously and to generate robust and comprehensive genetic information. Several updates of the Ion Torrent system may soon enable to detect also gene copy number alterations and translocations to more comprehensively cover the whole spectrum of genomic alterations refining the identification of reliable and reproducible biomarkers of response/resistance to the targeted treatment of CRC.

\section{Take home messages}

- Ion Torrent Personal Genome Machine (PGM), and the Ion AmpliSeq Colon and Lung Cancer Panel, enabled our laboratory to adopt next-generation sequencing.

- Less information is available on the uncommon mutated genes of the CRC genomic landscape.

- In a routine diagnostic setting, the AmpliSeq Colon and Lung Cancer Panel had the potential to generate robust and comprehensive genetic information.

\section{Handling editor Runjan Chetty}

Contributors UM, PP and GT conceived the study and wrote the paper. RS performed the experimental part. EV, GG and CB contributed as pathologists. CC and $\mathrm{MB}$ contributed as oncologists.

Competing interests None declared.

Patient consent Obtained.

Provenance and peer review Not commissioned; externally peer reviewed.
Open Access This is an Open Access article distributed in accordance with the Creative Commons Attribution Non Commercial (CC BY-NC 4.0) license, which permits others to distribute, remix, adapt, build upon this work non-commercially, and license their derivative works on different terms, provided the original work is properly cited and the use is non-commercial. See: http://creativecommons.org/ licenses/by-nc/4.0/

\section{REFERENCES}

1 Malapelle U, Carlomagno C, de Luca C, et al. KRAS testing in metastatic colorectal carcinoma: challenges, controversies, breakthroughs and beyond. J Clin Pathol 2014;67:1-9.

2 Douillard JY, Siena S, Cassidy J, et al. Randomized, phase III trial of panitumumab with infusional fluorouracil, leucovorin, and oxaliplatin (FOLFOX4) versus FOLFOX4 alone as first-line treatment in patients with previously untreated metastatic colorectal cancer: the PRIME study. J Clin Oncol 2010;28:4697-705.

3 Tops BB, Normanno N, Kurth $\mathrm{H}$, et al. Development of a semi-conductor sequencing-based panel for genotyping of colon and lung cancer by the Onconetwork consortium. BMC Cancer 2015;15:26.

4 Malapelle U, Vigliar E, Sgariglia R, et al. Ion Torrent next-generation sequencing for routine identification of clinically relevant mutations in colorectal cancer patients. J Clin Pathol 2015;68:64-8.

5 Cancer Genome Atlas Network. Comprehensive molecular characterization of human colon and rectal cancer. Nature 2012;487:330-7.

6 Wong NA, Gonzalez D, Salto-Tellez M, et al. RAS testing of colorectal carcinoma-a guidance document from the Association of Clinical Pathologists Molecular Pathology and Diagnostics Group. J Clin Pathol 2014;67:751-7.

7 Normanno N, Rachiglio AM, Lambiase M, et al. Heterogeneity of KRAS, NRAS, BRAF and PIK3CA mutations in metastatic colorectal cancer and potential effects on therapy in the CAPRI GOIM trial. Ann Oncol 2015;26:1710-4.

8 Chevrier S, Arnould L, Ghiringhelli F, et al. Next-generation sequencing analysis of lung and colon carcinomas reveals a variety of genetic alterations. Int J Oncol 2014;45:1167-74.

9 Weigelt B, Reis-Filho JS. Activating mutations in HER2: neu opportunities and neu challenges. Cancer Discov 2013;3:145-7.

10 Greulich H, Kaplan B, Mertins P, et al. Functional analysis of receptor tyrosine kinase mutations in lung cancer identifies oncogenic extracellular domain mutations of ERBB2. Proc Natl Acad Sci USA 2012;109:14476-81.

11 Kavuri SM, Jain N, Galimi F, et al. HER2 activating mutations are targets for colorectal cancer treatment. Cancer Discov 2015;5:832-41.

12 Williams CS, Bernard JK, Demory Beckler M, et al. ERBB4 is over-expressed in human colon cancer and enhances cellular transformation. Carcinogenesis 2015;36:710-18.

13 Kogita A, Yoshioka Y, Sakai K, et al. Inter- and intra-tumor profiling of multi-regional colon cancer and metastasis. Biochem Biophys Res Commun 2015;458:52-6.

14 Hechtman JF, Sadowska J, Huse JT, et al. AKT1 E17K in colorectal carcinoma is associated with BRAF V600E but not MSI-H status: a clinicopathologic comparison to PIK3CA helical and kinase domain mutants. Mol Cancer Res 2015;13:1003-8.

15 Lan YT, Jen-Kou L, Lin CH, et al. Mutations in the RAS and PI3K pathways are associated with metastatic location in colorectal cancers. J Surg Oncol 2015;111:905-10.

16 Day FL, Jorissen RN, Lipton L, et al. PIK3CA and PTEN gene and exon mutation-specific clinicopathologic and molecular associations in colorectal cancer. Clin Cancer Res 2013;19:3285-96.

17 Stachler MD, Rinehart E, Lindeman N, et al. Novel molecular insights from routine genotyping of colorectal carcinomas. Hum Pathol 2015;46:507-13.

18 Avizienyte $E$, Roth $S$, Loukola $A$, et al. Somatic mutations in LKB1 are rare in sporadic colorectal and testicular tumors. Cancer Res 1998;58:2087-90.

19 Liu Y, Marks K, Cowley GS, et al. Metabolic and functional genomic studies identify deoxythymidylate kinase as a target in LKB1-mutant lung cancer. Cancer Discov 2013;3:870-9.

20 Fearon ER. Molecular genetics of colorectal cancer. Annu Rev Pathol 2011;6:479-507.

21 Fleming NI, Jorissen RN, Mouradov D, et al. SMAD2, SMAD3 and SMAD4 mutations in colorectal cancer. Cancer Res 2013;73:725-35.

22 Zhang $B$, Zhang $B$, Chen $X$, et al. Loss of Smad4 in colorectal cancer induces resistance to 5-fluorouracil through activating Akt pathway. $\mathrm{Br} J$ Cancer 2014;110:946-57.

23 Alhopuro P, Alazzouzi H, Sammalkorpi $H$, et al. SMAD4 levels and response to 5-fluorouracil in colorectal cancer. Clin Cancer Res 2005;11:6311-6.

24 Akhoondi $S$, Sun D, von der Lehr N, et al. FBXW7/hCDC4 is a general tumor suppressor in human cancer. Cancer Res 2007;67:9006-12.

25 Bamford S, Dawson E, Forbes S, et al. The COSMIC (Catalogue of Somatic Mutations in Cancer) database and website. Br J Cancer 2004;91:355-8.

26 Wang Y, Liu Y, Lu J, et al. Rapamycin inhibits FBXW7 loss-induced epithelial-mesenchymal transition and cancer stem cell-like characteristics in colorectal cancer cells. Biochem Biophys Res Commun 2013;434:356-6. 
27 Yokobori T, Yokoyama Y, Mogi A, et al. FBXW7 mediates chemotherapeutic sensitivity and prognosis in NSCLCs. Mol Cancer Res 2014;12:32-7.

28 Jardim DL, Wheler JJ, Hess K, et al. FBXW7 mutations in patients with advanced cancers: clinical and molecular characteristics and outcomes with mTOR inhibitors. PLOS ONE 2014;9:e89388.
29 Bai J, Gao J, Mao Z, et al. Genetic mutations in human rectal cancers detected by targeted sequencing. J Hum Genet 2015;60:589-96.

30 Fender AW, Nutter JM, Fitzgerald TL, et al. Notch-1 promotes stemness and epithelial to mesenchymal transition in colorectal cancer. I Cell Biochem 2015;116:2517-27. 